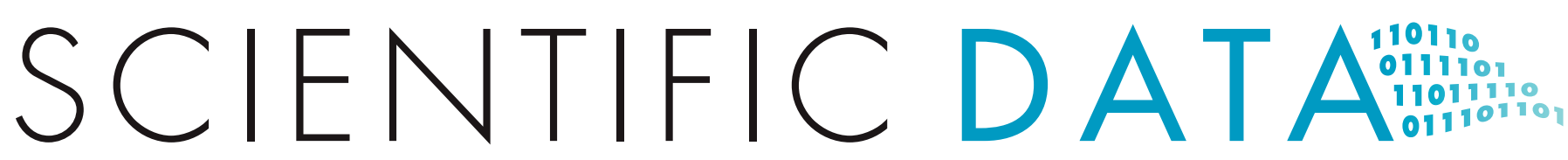

\title{
OPEN Data Descriptor: A collection of public transport network data sets for 25 cities
}

Received: 8 January 2018 Accepted: 20 March 2018

Published: 15 May 2018

\section{Rainer Kujala ${ }^{1}$, Christoffer Weckström ${ }^{2}$, Richard K. Darst ${ }^{1}$, Miloš N. Mladenović ${ }^{2}$ \& Jari Saramäki ${ }^{1}$}

Various public transport (PT) agencies publish their route and timetable information with the General Transit Feed Specification (GTFS) as the standard open format. Timetable data are commonly used for PT passenger routing. They can also be used for studying the structure and organization of PT networks, as well as the accessibility and the level of service these networks provide. However, using raw GTFS data is challenging as researchers need to understand the details of the GTFS data format, make sure that the data contain all relevant modes of public transport, and have no errors. To lower the barrier for using GTFS data in research, we publish a curated collection of 25 cities' public transport networks in multiple easy-to-use formats including network edge lists, temporal network event lists, SQLite databases, GeoJSON files, and the GTFS data format. This collection promotes the study of how PT is organized across the globe, and also provides a testbed for developing tools for PT network analysis and PT routing algorithms.

\begin{tabular}{|c|c|}
\hline Design Type(s) & data integration objective - source-based data transformation objective \\
\hline Measurement Type(s) & Public Transportation \\
\hline Technology Type(s) & digital curation \\
\hline Factor Type(s) & geographic location \\
\hline Sample Characteristic(s) & $\begin{array}{l}\text { City of Adelaide city Belfast City B Berlin Bordeaux } \bullet \text { Brisbane } \\
\text { Canberra City of Detroit Dublin Grenoble Helsinki } \bullet \text { Municipality } \\
\text { of Kuopio City of Lisbon Luxembourg City City of Melbourne } \\
\text { Nantes Palermo Commune of Paris City of Prague } \\
\text { Roma Rennes }\end{array}$ \\
\hline
\end{tabular}

${ }^{1}$ Department of Computer Science, Aalto University, P.O. Box 15400, FI-00076 Aalto/Espoo, Finland. ${ }^{2}$ Department of Built Environment, Aalto University, P.O. Box 14100, FI-0076 Aalto/Espoo, Finland. Correspondence and requests for materials should be addressed to R.K. (email: Rainer.Kujala@aalto.fi). 


\section{Background \& Summary}

Public transport (PT) networks help to provide efficient and sustainable mobility in cities around the world $^{1}$. PT network structure and schedules are challenging to plan, as one has to specify both the static structure of the network as well as the detailed schedules of PT vehicle departures. In particular, planners should have adequate tools for the analysis and optimization of the PT networks ${ }^{2-6}$, as well as expertise on how PT networks are structurally organized across cities ${ }^{7-12}$. For developing such tools and quantifying features of efficient public transport systems in different cities, detailed data on PT network operations are required.

Fortunately, an increasing number of public transport agencies publish their route and schedule data with the General Transit Feed Specification (GTFS, https://developers.google.com/ transit/gtfs/) as the standard, open format. GTFS specifies how to present PT service supply with a series of CSV (comma-separated-values) plain text files constituting a GTFS feed. GTFS data is primarily used for PT passenger routing, but it can also be used for research, for instance for modeling PT-provided accessibility ${ }^{4,6,13}$.

GTFS data are increasingly available through the web sites of PT agencies as well as online repositories such as Transitland (http://transit.land) and TransitFeeds (http://transitfeeds. com). However, using GTFS data for studying how PT is organized in different cities remains challenging. The first challenge is that the PT timetable data covering a city are often fragmented into multiple feeds, each describing the operations of a single PT operator. These multiple feeds must be merged to cover all relevant modes of transport in the area of interest. The second challenge is the opposite of fragmentation: sometimes feeds are provided for large areas, such as whole countries, and city-level data has to be filtered from the feeds spatially, which requires specifying city boundaries. The third challenge is that GTFS data may contain errors, e.g., in the coordinates of PT stops, or times of operations. Thus, the data have to go through a set of validation steps before they are used. The fourth and final challenge is that learning the details of the GTFS standard can be time-consuming and slow down the adoption of GTFS data; network scientists, for example, are much more familiar with other data formats.

The above challenges call for easy-to-use, validated data sets on urban public transport networks. However, to the best of the authors' knowledge, the only such data set is the multi-modal, temporal public transport network of Great Britain ${ }^{14}$. To this end, we have published a collection of PT network data sets for 25 cities in multiple, easy-to-use data formats. The locations of these cities, as well as examples of two computer-generated route maps are shown in Fig. 1. The provided formats include SQLite databases, GeoJSON-files usable for GIS-based analysis, static network edge lists and temporal network event lists commonly used by the network scientists, as well as the GTFS data format.

To compile this collection of PT networks, we first downloaded the relevant GTFS feeds for each of the 25 cities, and then imported these feeds into a database, sometimes merging feeds from multiple sources. Then, we checked the data for errors, and filtered the databases to match the spatial bounds of the cities. As GTFS feeds do not always contain information on transfer times between stops, we augmented the GTFS data with stop-to-stop walking distances using street network data from the OpenStreetMap project (http://wWw. openstreetmap.org, https://planet.osm.org).

The primary aim of publishing this collection of extracts is to facilitate comparative research on PT networks. However, the collection can be used for other purposes too. For instance, the city extracts can be used as a test bed for developing computational methods for PT network analysis and routing algorithms ${ }^{15}$. To make the collection of PT data sets easy to access, we have also launched an online repository http://transportnetworks.cs.aalto.f $i$ that allows interactive and visual exploration of the data.

\section{Methods}

For background, we introduce the structure and contents of a GTFS feed by listing the files in the feed and their contents in Table 1. As the different modes of PT transport, such as "bus" or "tram", are relevant in our data processing pipeline we list also their definitions, numeric codes, and short tag names in Table 2. For more detailed information on the structure of each file and how they relate to each other, please see https://developers.google.com/transit/gtfs/reference/.

Our data processing pipeline for producing the city extracts consists of multiple steps, as shown in Fig. 2. Each of these steps is detailed below.

\section{Step 1: Selecting cities and downloading source data}

When selecting the cities whose PT networks are to be included in our collection, we focused on covering cities of different sizes, from different continents, and from various geographies. The final selection of cities was, however, heavily affected by the availability of data, as many cities do not yet publish their data in the GTFS format. In addition, the licensing terms for the source data affected our selection of cities. Many cities and countries that publish GTFS data provide non-standard custom licenses that can be hard and time-consuming to interpret, licenses that do not allow modification or redistribution of the data, or no licensing information at all. Thus, for practical reasons and to guarantee free data reuse for scientific purposes, we have included only cities for which there was data available under public domain or one of the commonly used open data licenses, such as the Creative Commons -licenses or the Open Database 


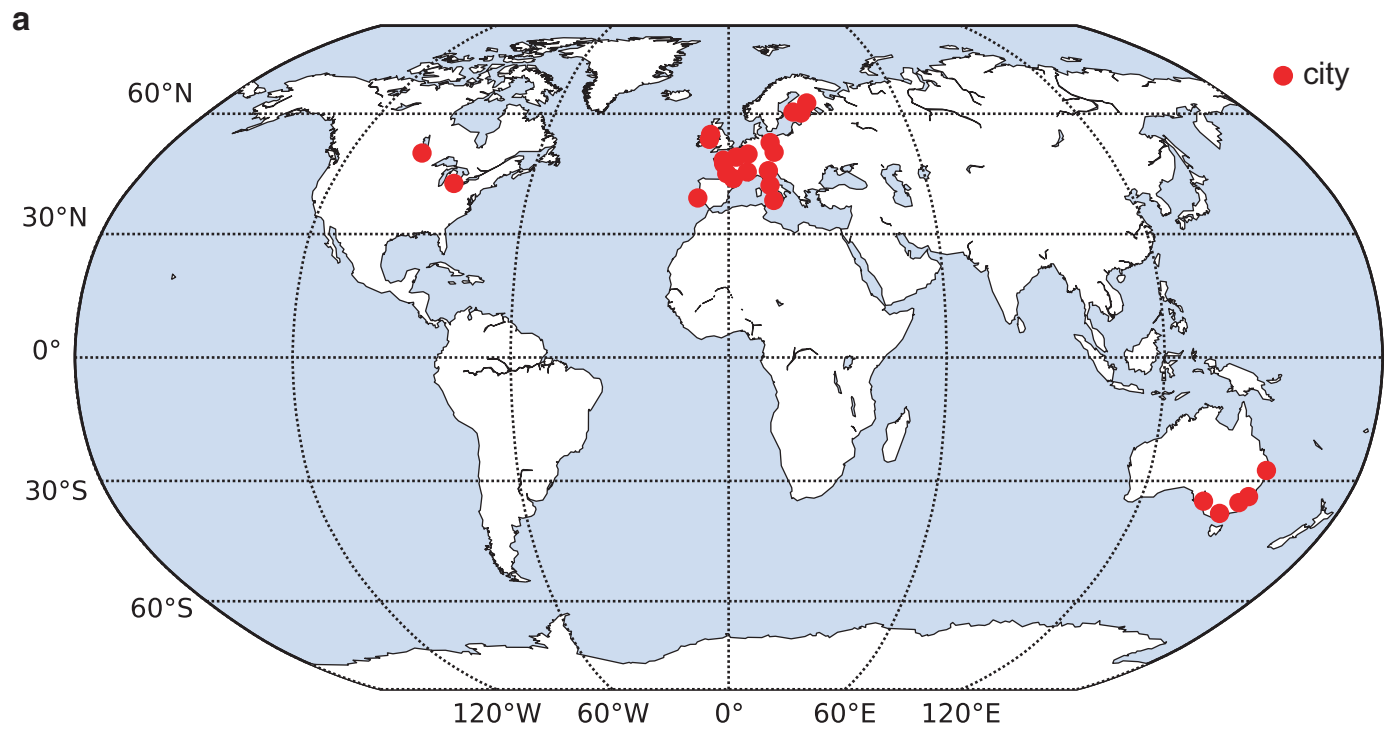

b

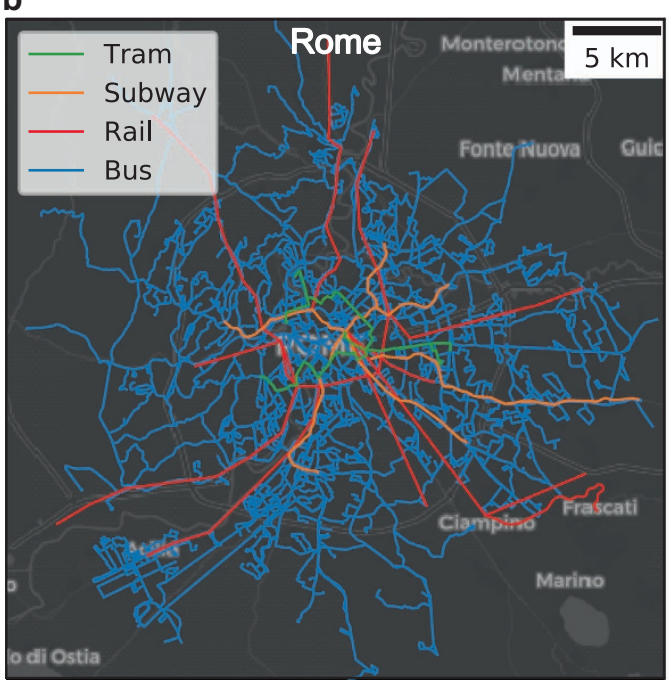

c

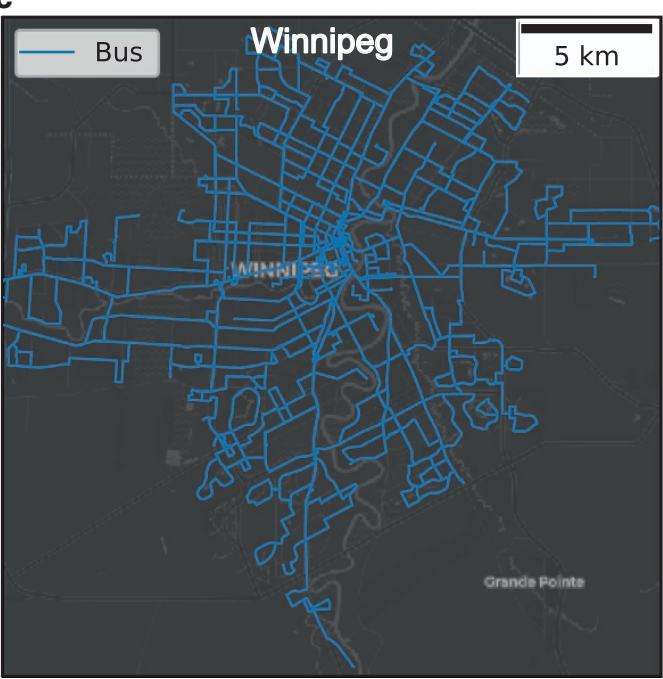

Figure 1. Spatial overview of the included cities, and two example PT network layouts. In panel a, we show the spatial overview of the cities' locations included in the collection. In panels $\mathbf{b}$ and $\mathbf{c}$, we show two example PT network layouts for the cities of Rome and Winnipeg. In Rome the PT network covers four different modes of travel (tram, subway, train, and bus), while the PT network of Winnipeg is operated solely based on buses. Background map courtesy of OpenStreetMap contributors, and Carto. Rome route network data used for visualization is published by Roma servizi per la Mobilità under CC BY 3.0 IT (https://creativecommons.org/ licenses/by/3.0/it).

License (ODbL) by the Open Data Commons. All these licenses allow redistributing the data and using it for scientific purposes. The exact licensing terms for the data of each city are provided alongside each data extract. The final list of the 25 selected cities is provided in Table 3 and these cities are visualized on the world map in Fig. 1.

In the process of discovering the GTFS data for each city, we searched the websites of PT agencies as well as known GTFS data repositories for the download URLs of the GTFS feeds. The data was then downloaded by Python scripts developed in-house that take care of possible authentication issues, store the downloaded data in a consistent manner on our premises, and allow for continuous re-downloading of the data on a weekly basis.

\section{Step 2: Importing data into SOLite databases}

In the second step, for each city, we imported the GTFS data into a relational SQLite database. An SQLite database is a single file that consists internally of different tables, each having their own sets of columns 


\begin{tabular}{|l|l|l|}
\hline Filename & Required & Defines \\
\hline agency.txt & Required & One or more transit agencies that provide the data in this feed. \\
\hline stops.txt & Required & Individual locations where vehicles pick up or drop off passengers. \\
\hline routes.txt & Required & Transit routes. A route is a group of trips that are displayed to riders as a single service. \\
\hline trips.txt & Required & Trips for each route. A trip is a sequence of two or more stops that occurs at specific time. \\
\hline stop_times.txt & Required & Times that a vehicle arrives at and departs from individual stops for each trip. \\
\hline calendar.txt & Required & $\begin{array}{l}\text { Dates for service IDs using a weekly schedule. Specify when service starts and ends, as well as days of the } \\
\text { week where service is available. }\end{array}$ \\
\hline calendar_dates.txt & Optional & $\begin{array}{l}\text { Exceptions for the service IDs defined in the calendar.txt file. If calendar_dates.txt includes ALL dates of } \\
\text { service, this file may be specified instead of calendar.txt. }\end{array}$ \\
\hline fare_attributes.txt & Optional & Fare information for a transit organization's routes. \\
\hline fare_rules.txt & Optional & Rules for applying fare information for a transit organization's routes. \\
\hline shapes.txt & Optional & Rules for drawing lines on a map to represent a transit organization's routes. \\
\hline frequencies.txt & Optional & Headway (time between trips) for routes with variable frequency of service. \\
\hline transfers.txt & Optional & Rules for making connections at transfer points between routes. \\
\hline feed_info.txt & Optional & Additional information about the feed itself, including publisher, version, and expiration information. \\
\hline
\end{tabular}

Table 1. The contents of a GTFS feed. Despite the .txt filename extensions, all files are comma-separatedvalues (CSV) files. This table is an excerpt from the "General Transit Feed Specification Reference" (https://developers.google.com/transit/gtfs/reference/) by Google LLC, licensed under http://creativecommons.org/licenses/by/3.0/CC BY 3.0.

\begin{tabular}{|l|c|l|c|}
\hline Mode tag & route_type & Description & Max. speed (km/h) \\
\hline tram & 0 & Tram, Streetcar, Light rail. Any light rail or street level system within a metropolitan area. & 100 \\
\hline subway & 1 & Subway, Metro. Any underground rail system within a metropolitan area. & 150 \\
\hline rail & 2 & Rail. Used for intercity or long-distance travel. & 300 \\
\hline bus & 3 & Bus. Used for short- and long-distance bus routes. & 100 \\
\hline ferry & 4 & Ferry. Used for short- and long-distance boat service. & 80 \\
\hline cablecar & 5 & Cable car. Used for street-level cable cars where the cable runs beneath the car. & 50 \\
\hline gondola & 6 & $\begin{array}{l}\text { Gondola, Suspended cable car. Typically used for aerial cable cars where the car is suspended from the } \\
\text { cable. }\end{array}$ & 50 \\
\hline funicular & 7 & Funicular. Any rail system designed for steep inclines. & 50 \\
\hline
\end{tabular}

Table 2. PT travel modes as defined by the GTFS standard. The data for "route_type" and "Description" columns are copied from the "General Transit Feed Specification Reference" (https://developers.google.com/ transit/gtfs/reference/) by Google LLC, licensed under CC BY 3.0 (http://creativecommons.org/licenses/by/3.0). The column "Mode tag" tells the tag used for different PT modes when producing single-mode network extracts. The column "Max. speed" tells the speed limit that we use to flag PT trips, where the average speed of trip distances is unrealistic.

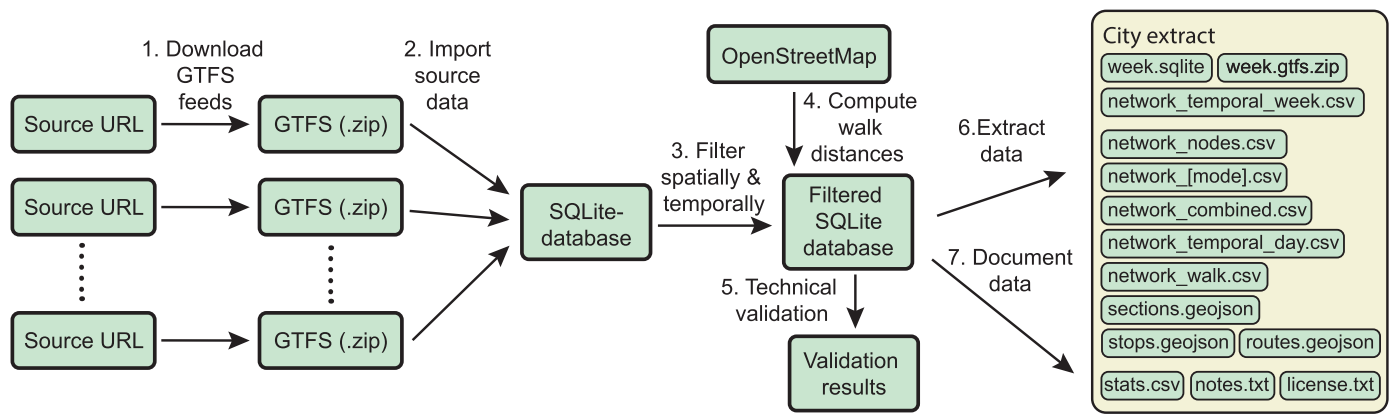

Figure 2. Data processing pipeline for one city. The pipeline for data processing consist of multiple subtasks. In the figure, the numbers indicate the order in which different tasks are carried out. Please see the main text for more details on each step and the final data formats. 


\begin{tabular}{|c|c|c|c|c|c|}
\hline City & Country & Stops & Links & Connections & License \\
\hline Adelaide & Australia & 7548 & 9257 & 404300 & CC BY 4.0 \\
\hline Belfast & Northern Ireland & 1917 & 2181 & 122693 & ODBL v1.0 \\
\hline Berlin & Germany & 4601 & 12079 & 1048218 & CC BY $3.0 \mathrm{DE}$ \\
\hline Bordeaux & France & 3435 & 4039 & 236595 & ODBL v1.0 \\
\hline Brisbane & Australia & 9645 & 11738 & 392805 & CC BY $3.0 \mathrm{AU}$ \\
\hline Canberra & Australia & 2764 & 3218 & 124305 & CC BY 4.0 \\
\hline Detroit & USA & 5683 & 5948 & 214863 & $\mathrm{CC} 0$ \\
\hline Dublin & Ireland & 4571 & 5567 & 407240 & CC BY 4.0 \\
\hline Grenoble & France & 1547 & 1682 & 114492 & ODBL v1.0 \\
\hline Helsinki & Finland & 6986 & 9072 & 686457 & CC BY 4.0 \\
\hline Kuopio & Finland & 549 & 704 & 32122 & CC BY 4.0 \\
\hline Lisbon & Portugal & 7073 & 8982 & 526179 & $\mathrm{CC} 0$ \\
\hline Luxembourg & Luxembourg & 1367 & 3234 & 186752 & $\mathrm{CC} 0$ \\
\hline Melbourne & Australia & 19493 & 21737 & 1098227 & CC BY 4.0 \\
\hline Nantes & France & 2353 & 2779 & 196421 & ODBL v1.0 fr \\
\hline Palermo & Italy & 2176 & 2561 & 226215 & CC BY 4.0 \\
\hline Paris & France & 11950 & 14781 & 1823872 & ODBL v1.0 fr \\
\hline Prague & Czechia & 5147 & 6754 & 670423 & $\mathrm{CC} 0$ \\
\hline Rennes & France & 1407 & 1671 & 109075 & ODBL v1.0 \\
\hline Rome & Italy & 7869 & 10206 & 1051211 & CC BY 3.0 IT \\
\hline Sydney & Australia & 24063 & 28815 & 1265135 & CC BY 4.0 \\
\hline Toulouse & France & 3329 & 3793 & 224516 & ODBL v1.0 \\
\hline Turku & Finland & 1850 & 2341 & 133512 & CC BY 4.0 \\
\hline Venice & Italy & 1874 & 2737 & 118519 & CC BY 3.0 IT \\
\hline Winnipeg & Canada & 5079 & 5846 & 333882 & PDDL \\
\hline
\end{tabular}

Table 3. The cities included in the collection, and basic information about their PT network

properties. The columns Stops, Links, and Connections indicate the number of each entity in the daily PT network extracts. The number of "Connections" corresponds to the number elementary, time-dependent PT vehicle movements between consecutive stops of a trip during the time span of the daily extract.

and rows. Due to the optimized structure and indexing, an SQLite database can be efficiently queried using Structured Query Language (SQL).

The table structure of the created SQLite database matches closely with the original tabular format of the data. Typically, each of the . txt files was imported into a table having the same name as the original file without the .txt extension. Additionally, to enable fast querying of the data, we created two convenience tables: table days enables querying the trips that run on each day, and table day_trips 2 enables fast discovery of trips that take place between two arbitrary time points expressed in Unix time. Furthermore, we created a new table, stop_distances, which lists the distances between stops that are at most 1,000 meters apart from each other, when measured using greatcircle distance. Last, we added a metadata table for storing auxiliary data on the feed as well as some pre-computed statistics.

When importing the data into a database, we changed any extended GTFS route types (describing the mode of travel) (https://developers.google.com/transit/gtfs/reference/ extended-route-types) into the standard set of route types (tram, subway, rail, bus, ferry, cable car, gondola, funicular) specified by the GTFS. This transformation was done in order to make comparisons across cities easier.

For faster querying of the data and storage efficiency, we replaced the original identifier columns containing strings $\left({ }^{*}\right.$ id) with columns $\left({ }^{*}\right.$ I $)$ having integer keys in all tables, except for the identifiers (shape_id) of the shapes.txt file. For instance, after the transformation, the main string identifier of each stop, stop_id, was replaced by an integer-valued column stop_I. However, the original string identifiers (*_id) were also retained in those tables where the _id field is used as the primary key mapping a row into a unique entity. Thus, for instance, in the stops table (describing the details of each PT stop) we preserved the stop_id column, but not in the stop_times table, as this data is excessively redundant.

Typically, the schedules for each PT route are specified by listing the stop times for each run of a route in the stop_times.txt file. However, sometimes the operations on a route are specified through the frequencies.txt file, providing the headway of a route but no departure times at the departure 


\begin{tabular}{|c|c|c|c|c|c|}
\hline City & Latitude & Longitude & $\mathrm{R}(\mathbf{k m})$ & Download date & Extract date \\
\hline Adelaide & -34.9213 & 138.5775 & 40 & 2016-12-07 & 2016-12-12 \\
\hline Belfast & 54.6001 & -5.9304 & 30 & $2017-10-30$ & 2016-09-05 \\
\hline Berlin & 52.5190 & 13.4029 & 30 & $2016-12-07$ & 2016-04-25 \\
\hline Bordeaux & 44.8412 & -0.5751 & 30 & 2016-12-07 & 2016-12-12 \\
\hline Brisbane & -27.4580 & 153.0226 & 40 & 2016-12-07 & 2016-12-12 \\
\hline Canberra & -35.2767 & 149.1254 & 30 & 2016-12-14 & 2017-01-09 \\
\hline Detroit & 42.3700 & -83.0807 & 30 & $2016-12-07$ & 2016-12-12 \\
\hline Dublin & 53.3497 & -6.2566 & 20 & 2016-12-07 & 2016-12-12 \\
\hline Grenoble & 45.1772 & 5.7228 & 20 & 2016-12-07 & 2016-11-14 \\
\hline Helsinki & 60.1733 & 24.9409 & 30 & $2016-12-07$ & 2016-12-12 \\
\hline Kuopio & 62.8945 & 27.6807 & 10 & 2016-12-07 & $2016-12-12$ \\
\hline Lisbon & 38.7096 & -9.1420 & 30 & 2017-01-30 & 2016-11-21 \\
\hline Luxembourg & 49.6111 & 6.1329 & 20 & 2016-12-07 & 2016-11-28 \\
\hline Melbourne & -37.8493 & 145.0793 & 50 & 2016-12-07 & 2016-12-12 \\
\hline Nantes & 47.2133 & -1.5516 & 20 & $2016-12-07$ & $2016-12-12$ \\
\hline Palermo & 38.1186 & 13.3598 & 20 & 2016-12-07 & 2014-09-22 \\
\hline Paris & 48.8619 & 2.3519 & 35 & $2016-12-07$ & 2016-12-12 \\
\hline Prague & 50.0846 & 14.4311 & 30 & 2016-12-07 & 2016-12-12 \\
\hline Rennes & 48.1079 & -1.6749 & 20 & $2016-12-07$ & 2016-12-19 \\
\hline Rome & 41.8963 & 12.4853 & 20 & $2017-10-25$ & 2017-11-06 \\
\hline Sydney & -33.8269 & 151.0643 & 50 & 2016-12-14 & 2016-12-19 \\
\hline Toulouse & 43.6021 & 1.4428 & 20 & $2016-12-07$ & $2016-12-12$ \\
\hline Turku & 60.4491 & 22.2671 & 10 & $2016-12-07$ & $2016-12-12$ \\
\hline Venice & 45.4882 & 12.2416 & 20 & $2016-12-07$ & $2016-12-12$ \\
\hline Winnipeg & 49.8819 & -97.1352 & 30 & 2016-12-07 & $2016-12-12$ \\
\hline
\end{tabular}

Table 4. Details of the spatial and temporal filtering parameters for each city. Columns "Latitude" and "Longitude" indicate the location of the defined city center point, and $R$ is the buffer radius used for spatial filtering.

stop. In this case, the travel durations between consecutive stops are nonetheless specified in the stop_times.txt. To unify the structure of the data in the SQLite database and the data extracts, we expanded such frequency-encoded route operations into multiple regular trips (route runs), resulting in additional rows in the trips, days, day_trips2 and stop_times tables.

If a city's PT operations were described in multiple feeds, we merged the feeds into a single database while prefixing all *_id columns separately for each feed. Otherwise, the data importing process progresses as usual.

For some cities, there were multiple stops with identical geospatial coordinates in the original data. To remove such duplicated stops, we aggregated together all stops that were less than one meter apart from each other. To do this, we first grouped the stops so that all stops that are less than one meter apart belong to the same group. Then, for each group, we selected one of the group's stops to represent all stops in the group. Finally, to remove the duplicated stops, the information on the other, non-representative stops were removed from stops and stop_distances tables, and their entries in stop_times were updated to use the representative stop's stop_I identifier.

While the SQLite imports are basically a direct import of the input CSV files, a researcher already benefits from them for multiple reasons. First, the import step converts semi-structured GTFS data into highly structured tabular data and catches any obvious inconsistencies. Second, different methods of representing the same thing (e.g. exact stop times vs. frequencies) are converted to a single, consistent representation. Third, SQLite is a common, open source format which can be used for ad hoc querying and data exploration much more easily than the original text files.

\section{Step 3: Spatial and temporal filtering of the data}

To ensure ease-of-access and consistency across the provided data extracts, we filter the SQLite databases both in space and time. The spatial filtering of the data is required for removing long-distance PT connections reaching far out from each city's urban area. Temporal filtering is performed for two reasons. First, we aim to cover typical operations, without any irregularities such as local public holidays that take place on working days. Second, given that PT time tables are commonly scheduled on a weekly basis, we select a representative one-week time period that should fully capture the provided PT services in a city, 


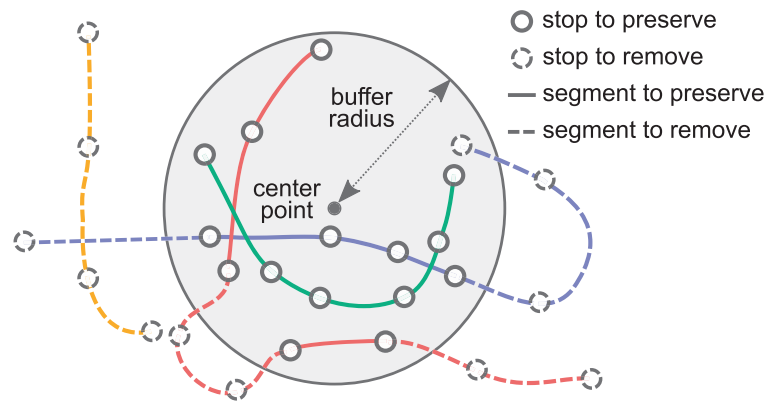

Figure 3. Spatial filtering of a GTFS feed based on stop coordinates. The city area is defined by a center point and an associated buffer radius. Only PT connections within the defined city area are included. Each line and color corresponds to a different PT trip (= one run of a PT route). When necessary, a trip is split into multiple parts so that only those parts of the trip are retained which run within the defined city area. Here, the red PT trip is split into two parts, as it temporarily exits and then re-enters the defined city area.

a

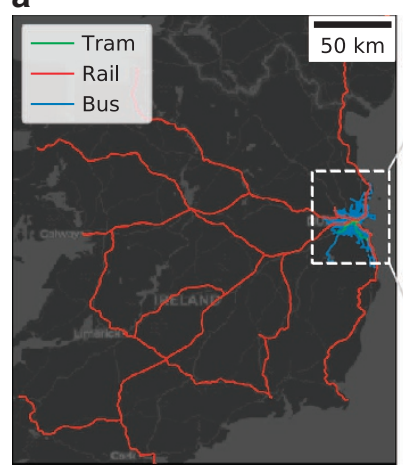

Routes before spatial filtering b

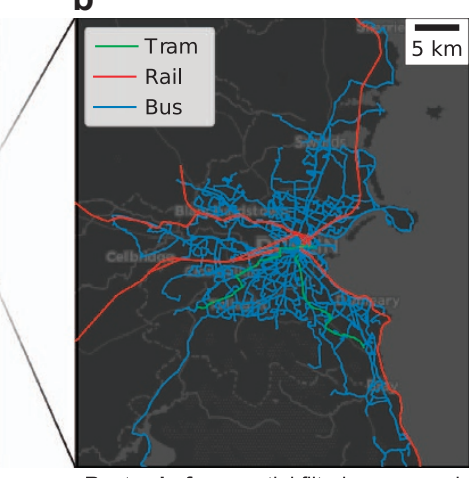

Routes before spatial filtering, zoomed



Routes after spatial filtering

Figure 4. The importance of spatial filtering. The figure shows the PT routes of Dublin's GTFS source data before and after the spatial filtering. In panels $\mathbf{a}$ and $\mathbf{b}$, we can see that the train routes present in one of the source feeds extend well beyond the city borders of Dublin. In panel c, we show the situation after spatial filtering, where the non-urban parts of the railway and bus networks have been removed. Note that, there are also some minor changes in the routes that reside within the buffer radius used for Dublin $(20 \mathrm{~km})$. These differences are not present in the actual data but are due to the visualization approach, which shows for each PT route the spatial shape of only one run of the route for efficiency reasons. Notably, the Dublin Area Rapid Transit (DART) route splits into two branches northeast from the city center. Due to the chosen visualization approach, this is now seen as a small discrepancy in the rail networks extending east from the city center, although this discrepancy is not present in the actual data.

as PT services are commonly planned to be week-periodic and variations in PT services across neighboring weeks are typically small. This also ensures consistency and comparability of results in future use of the data set, as the time-period of the data extracts are precisely defined. The final parameters used for spatial and temporal filtering of the data are provided in Table 4.

\section{Spatial filtering}

Spatial filtering of a GTFS feed is challenging for two reasons. First, the non-administrative areal limits of a city or metropolitan area can be difficult to define, and multiple different definitions exist ${ }^{16}$. Second, PT routes can span large areas, which needs to be taken into account when filtering the data.

While one could argue for different definitions of a city area, most likely no spatial filtering approach is ideal for all use cases. In our spatial filtering approach, we decided to pursue these goals: (i) limit the spatial extent of the city extracts to the approximate spatial bounds of the city, (ii) include only PT connections within the spatial bounds of the city, and (iii) cause minimal artifacts in the resulting data extracts, and the PT network structure, due to cutting of PT trips. 


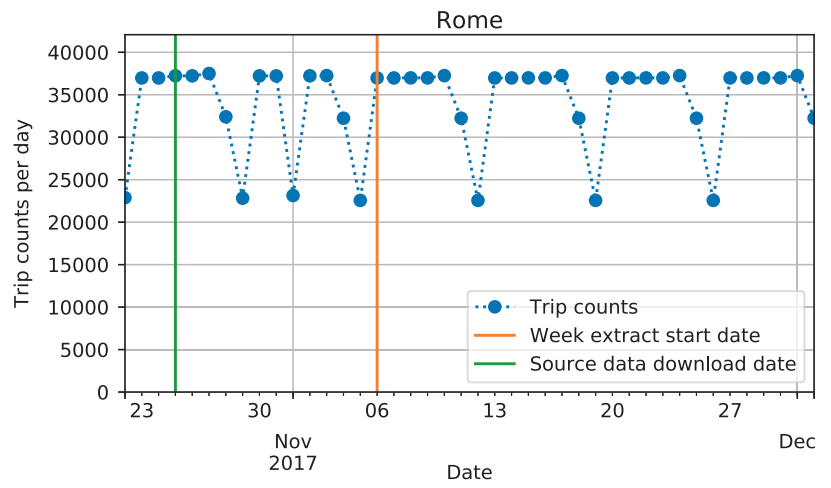

Figure 5. Temporal filtering is required for selecting a representative week and day. The daily variations in PT trip counts for Rome show the regular weekly pattern in the provided GTFS data. On 1st of November, the All Saints Day, we can see a drop in PT services due to the public holiday. Because of this, the next full week after All Saints' Day is selected as the representative week.

To this end, we adopted the following approach. First, we defined the extents of each city heuristically based on the stop locations and on satellite imagery. Note that while we could have also used administrative boundaries of the city, these may not properly reflect how the PT network is planned. For each city, we then selected a central point and a radius around this central point that should cover all the continuous and dense parts of the city and its PT network. The central point was selected to be approximately at the center of the city area, if the shape of the urban city area was approximately round. If the city area was very asymmetric, the central point was chosen to be at the city center, e.g. close to to the main railway center. Then we preserved all PT operations residing within this buffer area.

If a PT trip (= one run of a PT route) temporarily exited the buffer area, but later returned back, we split the trip into two or more subtrips, with each subtrip going through only stops that reside within the defined city area. In this case, the original trip is replaced in the SQLite database by the split trips, which were assigned a trip_id of the form [original_trip_id]_splitted_part_\{i\}, where $\{i\}$ stands for the ordinal number of the split trip. We chose to split trips that go outside the defined city area in order to prevent artifactual PT connections, which would have been emerged, e.g., if we would have only removed the stop time entries occurring outside the defined city area. We illustrate the spatial filtering approach in Fig. 3. An example where spatial filtering was especially necessary is showcased in Fig. 4

All trips that had only one entry in the stop_times table were removed from the trips table along with the corresponding entries in the stop_times table. Finally, all other tables' rows which are no longer "referred to" by any other tables are removed from the SQLite database.

\section{Temporal filtering}

The dates for the temporal filtering were chosen based on the number of daily trips of the included feeds. We ensured that there was a clear weekly periodicity in the feeds, so that working days have roughly the same number of trips and that weekends have a different schedule. Then we selected the dates for the week-long and day-long extracts, where we aimed for a typical work week without major exceptions in the PT operations, such as national holidays. To this end, we ensured that all weekdays have at least $90 \%$ of the maximum number of daily PT trips during the time span of the week-long extract. This enabled us to select the extract time spans in a semi-automatic manner, as illustrated in Fig. 5. If a city's timetable data consisted of multiple GTFS sources, we also ensured that each of the feeds was valid for the dates selected.

After the week- and day-extract start dates have been selected, our temporal filtering process preserves all PT trips in the GTFS data that depart within the precise timespan of the selected week or day. The precise time-span for the weekly extracts was set to range from 03:00:00 on Monday to 02:59:59 on the next Monday, and for the daily extracts from 03:00:00 on Monday 02:59:59 on the next day. We chose 3AM to be the "cutting point" as at this hour of day there were typically very few number of PT trip departures in all of the included cities. Note, that while the data contains no PT events before the beginning of the defined time-span, there can be PT events taking place after the end of the defined timespan, as some trips starting before the end of the time-span are still operating after the end of the time span.

In practice, the filtering process required removing entries from the calendar, calendar_dates, days, and day_trips 2 tables, and adapting the service information in the calendar, calendar_dates and trips tables for the preserved trips. Finally, all other tables' rows which are no longer being "referred to" by any other tables are removed from the SQLite database. 


\begin{tabular}{|c|c|}
\hline File & Description \\
\hline week.sqlite & An SQLite database covering the operations for a week. \\
\hline week.gtfs.zip & $\begin{array}{l}\text { A spatially and temporally filtered GTFS feed covering the operations taking place during a } \\
\text { week. }\end{array}$ \\
\hline network_temporal_week.csv & $\begin{array}{l}\text { Describes the PT operations on the level of elementary connections taking place during one full } \\
\text { week. }\end{array}$ \\
\hline network_nodes.csv & Information on the nodes for the network extracts. \\
\hline network_[mode].csv & $\begin{array}{l}\text { Static networks for each PT mode specified by the GTFS standard. Includes statistics for each } \\
\text { link computed based on the operations on a Monday. }\end{array}$ \\
\hline network_combined.csv & $\begin{array}{l}\text { A combined static network of all PT modes. Includes statistics for each link computed based on } \\
\text { the operations taking place on a Monday. }\end{array}$ \\
\hline network_walk.csv & $\begin{array}{l}\text { A combined static network of all PT modes. Includes statistics for each link computed based on } \\
\text { the operations taking place on a Monday. }\end{array}$ \\
\hline network_temporal_aay.csv & Describes the PT operations on the level of elementary connections taking place on a Monday. \\
\hline stops.geojson & Information on the nodes in GeoJSON format. \\
\hline sections.geojson & Each stop-to-stop section in GeoJSON format. \\
\hline routes.geojson & Public transport routes in GeoJSON format. \\
\hline stats.csv & Simple statistics describing the PT operations on a Monday. \\
\hline notes.txt & $\begin{array}{l}\text { Additional information such as the feeds' download dates, original source URLs, and time zone. } \\
\text { We describe here also specialties of the data set, such as missing modes of PT. }\end{array}$ \\
\hline license.txt & License information for the feed. \\
\hline$[\mathrm{x}]$ _legal_code.txt & Legal code for the provided data license. \\
\hline
\end{tabular}

Table 5. Data provided for each city. See the main text for more detailed description of each file.

Step 4: Computing walking distances between stops

As GTFS data format does not require one to provide transfer times or walking distances between stops, we computed stop-to-stop distances for each city to enable accurate modeling of walking transfers between stops. To limit the number of stops pairs, we computed stop-to-stop distances only between stops that were less than $1 \mathrm{~km}$ apart from each other. To this end, we used data from OpenStreetMap covering the whole planet (https://planet.osm.org/, OpenStreetMap contributors). For the actual routing, we used the open source GraphHopper routing library https://github.com/ graphhopper/graphhopper, and augmented the SQLite databases with these results. After the walk routing had been performed, all pairs of stops that were found unreachable by the OpenStreetMapbased pedestrian routing were removed from the stop_distances table. This would be the case e.g. when one of the two stops resided on an island, from which there is no bridge to the mainland, where the other stop resides. In few cases, where OpenStreetMap is lacking accurate data, the routing can cause PT stops to become unconnected in the walking network or the walking distance to become artificially large. However, the accuracy and coverage of OpenStreetMap data is typically so good that it can be used for reliable pedestrian and bike routing in public transport journey planners.

\section{Step 5: Data validation}

At this stage, we performed validation steps for the SQLite database. Please see Section "Technical Validation" for more information.

\section{Step 6: Creating extracts from the city databases}

As a final step of our pipeline, we extracted data from the database, and created the extracts described in Section "Data Records".

\section{Code availability}

Alongside the data, we share also our software and scripts for the full data processing pipeline. All code has been written for Python 3.5 and has been tested to work on both Mac and Linux. This code for downloading the original data and processing of the data into the city-sized extracts is available at https://github.com/CxAalto/gtfs_data_pipeline ${ }^{17}$. Internally, this pipeline heavily uses our in-house developed Python package gtfspy (https://github.com/CxAalto/ gtf $\mathrm{spy})^{18}$. The Java code for running the pedestrian routing is provided alongside the gtfspy Python package. Both software resources are available under the MIT license (https://opensource. org/licenses/MIT). 


\begin{tabular}{|c|c|c|c|c|c|c|c|c|c|c|c|c|c|c|c|c|c|}
\hline Extract & stop_I & latitude & longitude & stop_name & from_ttop_I & to_stop_I & n n-vehides & duration_avg & route___counts & route_type & d & d_walk & dep_time_ut & arr_time_ut & trip_I & route_I & routename \\
\hline network_nodes.csv & $\checkmark$ & $\checkmark$ & $\checkmark$ & $\checkmark$ & & & & & & & & & & & & & \\
\hline network_walk.csv & & & & & r &  & & & & & 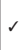 & s & & & & & \\
\hline network_[mode].csv & & & & & $\checkmark$ & s & r & s & 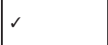 & & $\checkmark$ & & & & & & \\
\hline network_combined.csv & & & & & $\checkmark$ & $\checkmark$ & $\checkmark$ & 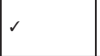 & $\checkmark$ & s & $\checkmark$ & & & & & & \\
\hline $\begin{array}{l}\text { network_temporal_day. } \\
\text { csv }\end{array}$ & & & & & $\checkmark$ & $\checkmark$ & & & & & & & o & or & lo & v & \\
\hline $\begin{array}{l}\text { network_temporal_week. } \\
\text { csv }\end{array}$ & & & & & $\checkmark$ & $\checkmark$ & & & & & & & 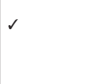 & s & s & $\checkmark$ & \\
\hline stops.geojson & 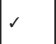 & 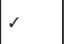 & $\checkmark$ &  & & & & & & & & & & & & & \\
\hline sections.geojson & & s & 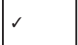 & 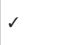 & s & s & s & s & s & s & & & & & & & \\
\hline routes.geojson & & 2 & v & & & & & & & o & & & & & & o & v \\
\hline
\end{tabular}

Table 6. Information contained by network and GeoJSON extracts.

\section{Data Records}

For each city, we provide the extracts listed in Table 5, which are then discussed in more detail. For long term archival, a bulk copy of all data is provided at Zenodo (Data Citation 1). In addition, we have also launched our own data repository http://transportnetworks.cs.aalto.fi that allows for interactive exploration and visualization of the data sets. Some of the provided data sets (week . sqlite, week.gtfs.zip, network_temporal_week.csv) cover the operations of the full week, while the rest cover operations during on typical working day (Monday).

\section{Week extracts}

- week.sqlite: The week.sqlite database is our master SQLite database for each city. This database has pre-built indices that allow efficient querying of data for many use cases. For more details on the structure of the SQLite database, see the Section "Step 2: Importing data into SQLite databases" under Methods.

- gtfs.zip: gtfs.zip is a GTFS export of the week.sqlite database covering the operations taking place during one week and has been spatially filtered to match the city bounds. Note that here any operations that were originally coded using the frequencies.txt file are expanded into the stop_times.txt files and thus no frequencies.txt file is present.

- network_temporal_week.csv: To allow easy investigation of the data using temporal network methodology ${ }^{19}$, we provide a week-long temporal network extract listing the elementary PT connections, or events, that describe the progression of a PT vehicle from a stop to its next stop along the route. Note that the rows in the file are not sorted by the departure time of the events, and that the values for trip_I repeat across the days. For more detailed description of the contents, see Table 6.

\section{Day extracts}

The network and GeoJSON extracts are aggregations of one working day (Monday). The precise descriptions of the contents of each file are provided in Table 6, and Table 7 describes data type for each data field. Below, we outline the content of each of the extracts.

Network extracts: All data related to the network extracts are provided as comma-separated-values files (.CSV) where semicolon (";") is used as the delimiter. Below we list the contents of each file.

- network nodes.csv:

Information on the PT stops that function as the nodes of the network.

- network_walk.csv:

This file describes the stop-to-stop walking distances between network nodes (stops). In the file we provide both euclidean (straight-line) distances and the values computed using Open Street Map routing. Note that links exist only between stop pairs where the straight-line distance $d$ is smaller than 1000 meters, and there is a footpath connection between the stops.

- network_[mode]. csv:

To enable easy investigation on the role of different PT modes, we provide a file network [mode] . CSV describing the operations for each mode of PT. Here, the [mode] part of the file name is one the values listed under the column "Mode tag" in Table 2. For instance, the tram network 


\begin{tabular}{|c|c|c|}
\hline Column & Data type & Explanation \\
\hline node_I & integer & The id used for a PT stop. \\
\hline latitude & float & Latitude expressed in WGS 84 coordinate system. \\
\hline longitude & float & Longitude expressed in the WGS 84 coordinate system. \\
\hline stop_name & string & Name of the stop as presented to PT passengers as presented in the original GTFS data. \\
\hline from_node_I & integer & From node's / stop's identifier. \\
\hline to_node_I & integer & To node's / stop's identifier. \\
\hline n_vehicles & integer & Number of PT vehicles that have traveled between two stops within a time interval. \\
\hline duration_avg & integer & Travel time between stops averaged over all PT vehicles rounded to one second accuracy. \\
\hline route_I_counts & list (string) & $\begin{array}{l}\text { A list of route_I's and the number of times each route has operated between two stops. For the network extracts, } \\
\text { this data is formatted as a string where each element is written as "route_I:count" and different routes are } \\
\text { separated by a comma. An example value for this field is thus " } 1: 3,2: 131,10: 93 \text { ". For the GeoJSON extracts, we } \\
\text { provide these values as part of the JSON object's attributes. Please note that the definition of a route varies across } \\
\text { the cities provided, and that routes can have deviations from their main paths for instance when traveling to and } \\
\text { from a depot. }\end{array}$ \\
\hline $\mathrm{d}$ & integer & Straight-line distance between two stops expressed in meters. \\
\hline d_walk & integer & Distance between two stops computed using OSM data expressed in meters. \\
\hline dep_time_ut & integer & $\begin{array}{l}\text { Departure time of an elementary PT connection in a temporal network expressed in Unix time (number of } \\
\text { seconds after 1.1.1970 00:00:00 UTC). }\end{array}$ \\
\hline arr_time_ut & integer & Arrival time of an elementary PT connection in a temporal network expressed in Unix time. \\
\hline trip_I & integer & Identifier for a trip. \\
\hline route_I & integer & Identifier for a route. \\
\hline route_name & string & Name of a route as shown to passengers. \\
\hline
\end{tabular}

Table 7. Explanations for fields used in the network and GeoJSON extracts.

\begin{tabular}{|l|l|}
\hline Variable name & Description \\
\hline n_stops & The number of stops used at least once. \\
\hline n_connections & Total number of elementary connections. \\
\hline n_links & $\begin{array}{l}\text { The total number of stop pairs between which at least one elementary PT } \\
\text { connection takes place. }\end{array}$ \\
\hline network_length_m & The sum of all links' (excluding walk) great circle distances, expressed in meters. \\
\hline link_distance_avg_m & Average link distance: network_length / n_links. \\
\hline vehicle_kilometers & Vehicle kilometers traveled. \\
\hline buffer_center_lat & Latitude of the center point used for spatial filtering. \\
\hline buffer_center_lon & Longitude of the center point used for spatial filtering. \\
\hline buffer_radius_km & The radius of the buffer circle used for the spatial filtering. \\
\hline extract_start_date & The starting date of the weekly extract, and the date used for the daily extracts. \\
\hline
\end{tabular}

Table 8. Contents of a stats.csv file describing PT operation statistics for one day.

of a city would be provided in file network_tram.csv. If there are no operations for a mode of $\mathrm{PT}$, then no network_[mode]. csv file is provided.

- network_combined.csv:

The file network_combined.csv contains the combined set of links of the mode-wise networks (network_[mode].csv). Note that there can be multiple links between two stops that correspond to different modes of travel, differentiated by the route_type field.

- network_temporal_day.csv:

Similarly to the network_temporal_week.csv, we provide a listing of temporal network events for the specified Monday matching the other Monday-related data extracts. Note that the rows in network_temporal_day.csv are not sorted by the departure time of the events. 
a



Excerpt from the official public transportation map in Helsinki b

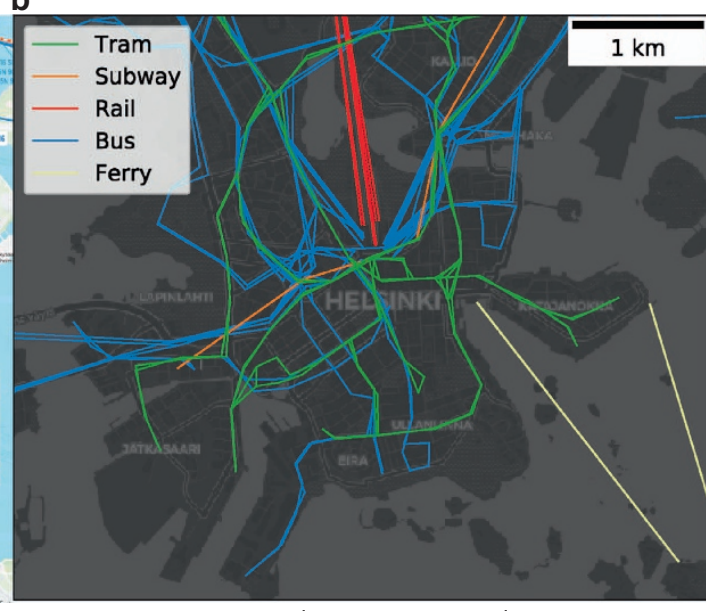

Map generated using static network extracts

Figure 6. Validating the coverage of the data extracts using publicly available PT route maps. In the figure, we compare the official route map of Helsinki to a computer-generated map created based on the static network extract for Helsinki. In panel a, we show an excerpt of the official PT transportation map of Helsinki city center published in early 2018 (routes and layout: Helsinki Region Traffic, background map OpenStreetMap). The blue, green, orange, and purple lines correspond to bus, tram, subway, and train routes, respectively. In panel $\mathbf{b}$, we show the computer-generated map used for validation purposes (background map OpenStreetMap). Note that on the computer-generated map, the lines do not follow the actual paths taken by the PT vehicles, but connect the two stops directly. By comparing the two maps, we can see that both maps contain same modes of PT and most PT lines can be found in both maps. However, given that the maps cover slightly different time frames, there are also some differences in the maps due to changes in the PT network. One such example is the new continuation of the subway line east from the city center.

GeoJSON extracts: To enable the data to be used in popular GIS-software and web-mapping tools, we provide also GeoJSON files for the stops, sections (i.e. PT network links) between the stops, and routes. All coordinates in the GeoJSON files are expressed in the WGS84 coordinate system.

- stops.geojson:

Information on the public transport stops.

- sections.geojson:

All stop-to-stop sections with data on operations.

- routes.geojson:

In this file we provide the stop coordinates for each route. Here, a distinct route is specified by the GTFS standard using the field route_id. Note that even if a route would operate in two directions, only one direction of the route is included. Also, the GTFS standard allows routes to go through different stops on different trips. Here, we provide the coordinates of stops of the trip that has the longest scheduled duration.

\section{Metadata}

license.txt

The licensing terms of each city extract are described in license.txt -files. The legal code of the referenced licenses are provided in an additional file $[x] \_l$ egalcode.txt, where $[x]$ stands for an abbreviation of the license name under which the data is provided.

notes.txt

In notes.txt we state how comprehensively the each city extract covers the PT services available within the city area. Sometimes we also provide minor additional remarks regarding the feed.

stats.csv

The statistics file containing the following summarizing the operations taking place on a Monday. The details of the provided statistics are listed in Table 8. 


\section{Technical Validation}

Our validation process consists of two major steps: automated data validation through various sanity checks and comparison of the data to openly available information on the PT network structures. The first step of our automated data validation is to check that the importing of the GTFS files into SQLite databases has been successful and there are no missing data.

For detecting actual errors in the schedule data, we computed for each city the number of cases (in the week.sqlite database) where:

1. distance between consecutive PT stops on a trip is longer than $20 \mathrm{~km}$;

2. five or more consecutive stops on a trip have the same scheduled arrival time;

3. trip duration is longer than 2 hours;

4. travel duration between consecutive stops is larger than 30 minutes;

5. the average speed of a PT trip is unrealistically high with respect to the PT mode in question. The mode-wise threshold speeds used for detecting unrealistically fast PT trips are listed in Table 2.

Most feeds raise warnings for at least some of these features. For instance, it is typical that five or more consecutive stops along a trip have been sloppily scheduled to have same arrival time, when the PT vehicle is heading towards its depot. Sometimes, on the other hand, these warnings are simply false alarms. Regardless whether such warnings are due to errors in the source data or are just peculiarities of the city's PT operations, researchers using the data should be aware of them. Therefore, we report the numbers of the above-issued, computer-generated warnings in file week_db_timetable_warnings_summary.log.

After the creation of the data extracts, we perform a visual sanity check of the data for each city. At this stage, we visualize all PT routes of each city on top of a map using an associated web-mapping tool (https://github.com/CxAalto/gtfspy-webviz) and assess whether the locations of the $\mathrm{PT}$ routes seem viable with respect to the surrounding land use, and that the spatial filtering process has been successful. In addition, we use the web-mapping tool to animate the movement of PT vehicles in time, and check that there are no striking peculiarities in the data, such as a PT vehicles suddenly jumping between distant locations.

Finally, we compare the computer-generated PT network visualizations to the publicly accessible information about the PT network and operations provided in each city, such as network maps and timetables. This process is illustrated in Fig. 6 where the official PT route map of Helsinki and a computer-generated route map based on the static PT network extracts are compared. Out of the 25 cities, we found all relevant urban public transport modes for 14 cities (Adelaide, Berlin, Brisbane, Canberra, Dublin, Helsinki, Kuopio, Luxembourg, Melbourne, Paris, Rome, Turku, Sydney, Winnipeg). For 9 cities (Belfast, Bordeaux, Grenoble, Nantes, Palermo, Prague, Rennes, Toulouse, Venice) only commuter trains or regional buses are missing from the data. For Detroit and Lisbon, services in a part of the city area are missing. For Lisbon data on the tram network is also missing. We report the above-listed cases in the notes.txt - file provided alongside each city extract.

\section{Usage Notes}

The spatially and temporally filtered GTFS data can be analyzed using many pieces of software. Of these, we mention our in-house-developed Python package gtfspy which was the core software block for creating these data extracts, and is interoperable with the provided week.sqlite databases. The package provides methods e.g. for computing various PT network statistics, as well as performing routing and accessibility analyses on the network.

The GeoJSON extracts can be easily analyzed and visualized using GIS-software such as QuantumGIS, as well as various web mapping tools.

Regarding the network data formats, there are multiple network analysis libraries available, such as the popular networkx library (http://networkx.github.io/) that can be directly used for analyzing the data. Note that when using these data for network analyses, the nodes (PT stops) have been minimally spatially aggregated. Thus, e.g. large bus or metro stations with multiple platforms often result in multiple network nodes, which should be taken into account especially when performing static network analyses.

\section{References}

1. Banister, D. The sustainable mobility paradigm. Transport Policy 15, 73-80 (2008).

2. Coffey, C., Nair, R., Pinelli, F., Pozdnoukhov, A. \& Calabrese, F. Missed connections: quantifying and optimizing multi-modal interconnectivity in cities. Proc. of the 5th ACM SIGSPATIAL International Workshop on Computational Transportation Science, 26-32 (2012)

3. Farahani, R. Z., Miandoabchi, E., Szeto, W. Y. \& Rashidi, H.. A review of urban transportation network design problems. Eur. J. Oper. Res. 229, 281-302 (2013).

4. Farber, S. \& Fu, L. Dynamic public transit accessibility using travel time cubes: Comparing the effects of infrastructure (dis) investments over time. Comput. Environ. Urban Syst. 62, 30-40 (2017).

5. Fayyaz, S. K., Liu, X. C. \& Zhang, G. “An efficient General Transit Feed Specification (GTFS) enabled algorithm for dynamic transit accessibility analysis”. PLoS ONE 12, e0185333 (2017)

6. Kujala, R., Weckström, C., Mladenovic, M. N. \& Saramäki, J. Travel times and transfers in public transport: Comprehensive accessibility analysis based on Pareto-optimal journeys. Comput. Environ. Urban Syst. 67, 41-54 (2018). 
7. Sen, P. et al. Small-world properties of the Indian railway network. Phys. Rev. E 67, 036106 (2003).

8. Sienkiewicz, J. \& Holyst, J. A. Statistical analysis of 22 public transport networks in Poland. Phys. Rev. E 72, 046127 (2005).

9. Von Ferber, C., Holovatch, T., Holovatch, Y. \& Palchykov, V. Public transport networks: empirical analysis and modeling. Eur. Phys. J. B 68, 261-275 (2009).

10. Derrible, S. \& Kennedy, C. The complexity and robustness of metro networks. Physica A 389, 3678-3691 (2010).

11. von Ferber, C., Berche, B., Holovatch, T. \& Holovatch, Y. A tale of two cities: Vulnerabilities of the London and Paris transit networks. J. Transp. Secur 5, 199-216 (2012).

12. Gallotti, R. \& Barthelemy, M. Anatomy and efficiency of urban multimodal mobility. Sci. Rep 4, 6911 (2014).

13. Salonen, M. \& Toivonen, T. Modelling travel time in urban networks: comparable measures for private car and public transport. J. Transp. Geogr. 31, 143-153 (2013).

14. Gallotti, R. \& Barthelemy, M. The multilayer temporal network of public transport in Great Britain. Sci. Data 2, 140056 (2015).

15. Bast, H. et al. Route planning in transportation networks in Algorithm Engineering: Selected Results and Surveys, 19-80 (Springer International Publishing, 2016).

16. Eurostat (European Union). Methodological manual on city statistics http://doi.org/10.2785/708009 (2017).

17. Kujala, R., Weckström, C. \& Darst, R. Code used for producing: "A collection of public transport network data sets for 25 cities". Zenodo https://doi.org/10.5281/zenodo.1186238 (2018).

18. Kujala, R. et al. Source code of the gtfspy Python package used for producing a collection of public transport network data sets. Zenodo https://doi.org/10.5281/zenodo.1186239 (2018).

19. Holme, P. \& Saramäki, J. Temporal networks. Phys. Rep 519, 97-125 (2012).

\section{Data Citation}

1. Kujala, R., Weckström, C. \& Darst, R. Zenodo https://doi.org/10.5281/zenodo.1186215 (2017).

\section{Acknowledgements}

The computational resources provided by the Aalto Science IT project are acknowledged. All authors thank the support from the Academy of Finland through the DecoNet project (No. 295499). In addition, this research has received partial support from BEMINE project (No. 303538). The authors thank Helsinki Region Transport for allowing to use their route maps as a part of this paper illustrations.

\section{Author Contributions}

R.K., C.W., R.D. wrote the code used for downloading the source data for the selected cities and transforming the data into the published extracts. All authors participated in the design of the data formats and in writing the paper.

\section{Additional Information}

Competing interests: The authors declare no competing interests.

How to cite this article: Kujala R. et al. A collection of public transport network data sets for 25 cities. Sci. Data 5:180089 doi: 10.1038/sdata.2018.89 (2018). The authors thank Helsinki Region Transport for allowing to use their route maps as a part of this paper illustrations.

Publisher's note: Springer Nature remains neutral with regard to jurisdictional claims in published maps and institutional affiliations.

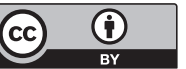

Open Access This article is licensed under a Creative Commons Attribution 4.0 International License, which permits use, sharing, adaptation, distribution and reproduction in any medium or format, as long as you give appropriate credit to the original author(s) and the source, provide a link to the Creative Commons license, and indicate if changes were made. The images or other third party material in this article are included in the article's Creative Commons license, unless indicated otherwise in a credit line to the material. If material is not included in the article's Creative Commons license and your intended use is not permitted by statutory regulation or exceeds the permitted use, you will need to obtain permission directly from the copyright holder. To view a copy of this license, visit http://creativecommons. org/licenses/by/4.0/

The Creative Commons Public Domain Dedication waiver http://creativecommons.org/publicdomain/ zero/1.0/ applies to the metadata files made available in this article.

(C) The Author(s) 2018 